\title{
Prevalence of Endometriosis in Infertile and Sub- Fertile Women
}

\author{
Shaista Zubair ${ }^{1 *}$, Hafsa Sheikh ${ }^{2}$, ShereenZulfiqarButta ${ }^{3}$, SamiaShuja $^{3}$ \\ ${ }^{1}$ Specialist Obstetrics and Gynecology Oasis Hospital, Abu Dhabi United Arab Emirates. \\ ${ }^{2}$ College of physician and surgeons of Pakistan \\ ${ }^{3}$ Jinnah Postgraduate Medical Centre Karachi Pakistan
}

Received: February 02, 2017; Accepted: February 09, 2017; Published: October 06,2017

*Corresponding author: Shaista Zubair, MRCOG.UK, MRCPI. Ireland, FCPS, MCPS, Specialist Obstetrics and Gynecology, Oasis Hospital Abu Dhabi, United Arab Emirates, Tel:+971 7401617; E-mail: shaistazubair212@gmail.com

\begin{abstract}
Introduction: Endometriosis is defined as the presence of functioning endometrial tissue at a site outside the uterus. It is a common and debilitating condition, diagnosis has traditionally been by laparoscopy.
\end{abstract}

Objective: To determine the frequency of endometriosis in infertile and sub- fertile women.

Study design: Descriptive cross - sectional study was carried out at the Department of Obstetrics and Gynecology, Jinnah postgraduate Medical Centre, Karachi, Pakistan (tertiary care hospital listed in WHO directory).

Methods: Patients of reproductive age were selected on convenient sampling technique, with history of infertility and sub-fertility with normal semen analysis of husband. Those with suspicious of cyst, Polycystic Ovary Syndrome and contraindication to laparoscopy were excluded.

Result: The frequency of endometriosis in women with infertility and sub-fertility was found to be $10(20 \%)$. We found no association between demographic features age, parity and socio-economic class) and occurrence of endometriosis.

Conclusion: Through this study we conclude that women with history of sub-fertility and infertility at a risk of endometriosis and must be subjected to diagnostic laparoscopy after excluding male factor contribution. Endometriosis is a progressive disease and the longer the diagnostic delay more advanced is the disease at the time of laparoscopy. Hence an early diagnostic laparoscopy in the evaluation of infertile women helps to diagnose endometriosis in its early stages and also reduces the numbers of patients of unexplained infertility.

Key words: Endometriosis; Infertility; Sub-Fertility; Laparoscopy

\section{Introduction}

Endometriosis is defined as the presence of endometrial-like tissue outside the uterus, which induces a chronic, inflammatory reaction. While some women with endometriosis experience painful symptoms and/or infertility, others have no symptoms at all. The exact prevalence of endometriosis is unknown but estimates range from 2 to $10 \%$ within the general female population but up to $50 \%$ in infertile women. [1] although endometriosis can be diagnosed incidentally in asymptomatic individual, it more commonly present with chronic or cyclical pelvic pain, sub-fertility or adnexal masses.[2]

Within this restricted case definition, the relation between endometriosis and infertility status is complex. At baseline, the prevalence of infertility (defined as attempting to become pregnant for $>1$ year without success) was greater among women with laparoscopic confirmation (20\%) than among those who were diagnosed without laparoscopic confirmation (4\%) potentially resulting in over sampling those with asymptomatic disease, approximately twenty percent of all infertile women are found to have endometriosis. [3]

Endometriosis is assumed to be one of the causative factors of infertility, although the mechanism remains to be elucidated mechanical factors distorting the pelvic anatomy and /or changes in the biochemical and cellular environment in the peritoneal cavity are possible contributing factors impairing fertility. Laparoscopy is a widely used diagnostic and therapeutic means of treating endometriosis associated infertility. [4]Timely diagnoses by early laparoscopy can reduce the complication and mortality that resulted due to delay in diagnosis of these diseases.[5]Laparoscopy remains the gold standard in evaluating an infertile female. [6] Infertility is the most frequent indication for laparoscopy to detect endometriosis. Without a diagnostic laparoscopy and hysteroscopy in selected patients infertility work up remains incomplete. [7]

Considering the current burden of endometriosis, the diagnostic challenges faced by gynecologists and the paucity of local data, the study aimed to calculate the frequency of endometriosis in women who underwent diagnostic laparoscopy in infertility and sub-fertility.

\section{Material and Method}

This hospital based descriptive cross sectional study was carried out at the department of Obstetrics and Gynecology, Jinnah postgraduate Medical Centre Karachi Pakistan from November-2006 to September 2007.A total of fifty patients via 
non probability convenient sampling technique were selected from outpatient department.

The women of ages 18-40 years, with history of primary and secondary infertility the mean parity of patients was $2.50 \pm$ 1.5(range: 0-3) included whose husband semen analysis were normal and women with normal luteal phase progesterone $>30$ $\mathrm{nmol} / \mathrm{L}$ were included. Socioeconomic status was based on the family basic earning.

Women with any an adulatory disorders e.g. Polycystic ovarian syndrome etc. Were excluded.

All the laparoscopies were done using standard procedure in Gynecology operation theatre. The presence of classical powder burn, blue/ black implant, vesicular hemorrhagic lesions, nodular, discolored lesions. Chocolate ovarian cyst, sub ovarian or peritoneal adhesions were all taken as evidence of endometriosis.

Endometriosis thus diagnosed was scored and classified according to revised American Fertility Society Classification.

Data was analyzed by using SPSS version 20. Age and parity was presented as mean +/-SD. Other variables like endometriosis and factors were presented as frequency and percentages. Data was stratified with respect to age, parity, type of infertility and socioeconomic status. Post-stratification Chi-square test is applied to observe the significance. P-value $\leq 0.05$ is taken as significant.

\section{Result}

Out of all women presenting with infertility and sub-fertility a total of 50 women underwent diagnostic laparoscopy. Of these, a total of $10(20 \%)$ women were found to have endometriosis based

\begin{tabular}{|c|c|c|}
\hline & \multicolumn{2}{|c|}{$(n=50)$} \\
\hline & mean(SD) & range \\
\hline Age & $28.7(5.45)$ & $(20-40)$ \\
\hline \multirow[t]{2}{*}{ Parity } & $2.50(1.5)$ & $(0-3)$ \\
\hline & frequency & percentage \\
\hline \multicolumn{3}{|c|}{ Type of infertility } \\
\hline Primary & 35 & $70 \%$ \\
\hline Secondary & 15 & $30 \%$ \\
\hline \multicolumn{3}{|c|}{ Endometriosis } \\
\hline Yes & 10 & $20 \%$ \\
\hline No & 40 & $80 \%$ \\
\hline \multicolumn{3}{|c|}{ Stages of endometriosis } \\
\hline Stage-I & 2 & $20 \%$ \\
\hline Stage-II & 4 & $40 \%$ \\
\hline Stage-III & 2 & $20 \%$ \\
\hline Stage-IV & 2 & $20 \%$ \\
\hline Total & 10 & $100 \%$ \\
\hline
\end{tabular}

on laparoscopic evidence. The mean age of patients was $28.7 \pm$ 5.45 years (range: 20-40years) and the majority of patients fell between ages 20 to 30 years. The mean parity of patients was $2.50 \pm 1.5$ (range: $0-3$ ).

The majority of patients in the study presented with primary infertility $35(70 \%)$ and rest of them presented with secondary infertility15 (30\%). Frequency of each stage of endometriosis were found to be $2(20 \%)$ for stage I, $4(40 \%)$ for stage II, $2(20 \%)$ for stage III and 2 (20\%) for stage IV. Descriptive statistics of age, parity, type of infertility, endometriosis and its stages has been summarized in Table 1.

Association between age, parity, tubal status, type of infertility and socio economic status via diagnostic laparoscopy has been summarized in Table 2. Statistically non-significant association was seen between age and endometriosis ( $p$-value=0.85)

Table2: stratification of endometriosis with regards to effect modifiers

\begin{tabular}{|c|c|c|c|c|}
\hline \multirow{3}{*}{ Variables } & \multicolumn{4}{|c|}{$(n=50)$} \\
\hline & \multicolumn{2}{|c|}{ Endometriosis } & \multirow{2}{*}{ Total } & \multirow{2}{*}{ P-Value } \\
\hline & Yes & No & & \\
\hline \multicolumn{5}{|c|}{ Age } \\
\hline $20-25$ years & 4 & 13 & 17 & \multirow{4}{*}{0.85} \\
\hline 26-30 years & 4 & 14 & 18 & \\
\hline 31-35 years & 1 & 9 & 10 & \\
\hline $36-40$ years & 1 & 4 & 5 & \\
\hline \multicolumn{5}{|c|}{ Parity } \\
\hline Null Para & 8 & 27 & 35 & \multirow{4}{*}{0.86} \\
\hline 1 Para & 1 & 5 & 6 & \\
\hline 2 Para & 1 & 7 & 8 & \\
\hline 3 Para & 0 & 1 & 1 & \\
\hline \multicolumn{5}{|c|}{ Tubal Status } \\
\hline Both tubes patent & 4 & 25 & 29 & \multirow{4}{*}{0.25} \\
\hline One tube block & 3 & 3 & 6 & \\
\hline $\begin{array}{l}\text { Tube patent covered with } \\
\text { adhesions }\end{array}$ & 1 & 4 & 5 & \\
\hline Both tubes block & 2 & 8 & 10 & \\
\hline \multicolumn{5}{|c|}{ Type Of Infertility } \\
\hline Primary & 8 & 27 & 35 & \\
\hline Secondary & 2 & 13 & 15 & 0.7 \\
\hline \multicolumn{5}{|c|}{ Socioeconomic Status } \\
\hline Lower class & 3 & 21 & 24 & \multirow{4}{*}{0.16} \\
\hline Lower middle & 4 & 41 & 45 & \\
\hline Upper middle & 2 & 5 & 7 & \\
\hline Upper & 1 & 0 & 1 & \\
\hline
\end{tabular}


Parity showed non-significant difference with ( $\mathrm{p}$-value $=0.86)$. Tubal status distribution presented as, out of 10 patients in 4 $(14.28 \%)$ patients both tubes were patent. 3 cases were with single tube blockage, where as in one case (25\%) both tubes were patent but they were covered with adhesion. Bilateral tubal blockade found in 2 cases $18.18 \%$ of cases stage VI. Not found any significant difference, status of tubes in association with endometriosis ( $p$-value $=0.25$ ).Structural damage to ovaries and tubes is therefore not responsible for infertility in cases of minimal and mild diseases, In contrary patients in stage IV had bilateral tubal blockade .Structural damage can be the reason of infertility in stage III and IV. Type of infertility showed onsignificant association with endometriosis with ( $p$-value $=0.70$ ) .data also stratified for socioeconomic classes and found to be no statistically significant difference with ( $p$-value $=0.16$ ).

\section{Discussion}

Endometriosis is a significant health problem for women in reproductive age. Endometriosis remains a difficult clinical problem due to its variable presentation, costly diagnosis and management. The true prevalence of endometriosis in the general population cannot be determined as it is impractical to subject asymptomatic general population to a surgical procedure. The present study found the frequency of endometriosis in infertile and sub-fertile patients to be $20 \%$ in our study 50 patients were included who presented with either primary or secondary infertility. Whereas laparoscopy was carried out for diagnostic purpose. Which reveal that $20 \%$ of such patients have endometriosis associated with infertility. The clinical manifestation of endometriosis is versatile and unpredictable in its presentation and course. While the association between endometriosis and infertility remains the subject of considerable debate. [8]

For a definite diagnosis of endometriosis, visual inspection of the pelvis at laparoscopy is the "gold standard" investigation. Unless disease is visible in vaginal inspection or else. Whereas it is proven by the result of the study that it should be performed in all suspected cases to confirm the diagnosis. Current study was conducted to discover the frequency of endometriosis in relation to infertility and sub-fertility. It is difficult to make a final statement about the incidence of disease even by experienced laparoscopist with vast experience of management of endometriosis $7 \%$ of the cases are missed and 50\% are underdiagnosed. Endometriosis occur in $7-10 \%$ of women in the general population and up to $50 \%$ of premenopausal women with a prevalence of $38 \%$ range 20 to $50 \%$ in infertile women. [8] The result of our study is not only supported by above mentioned statement but also by another study conducted by Tarek A et al, in Egypt has similar results. [9]This is also supported by study Nakagawak et al, (2005) their study shows $21 \%$ incidence of endometriosis.[10]

Regarding the age distribution majority of patients with infertility and endometriosis presented between 20 to 30 years of age and same age distribution found in the study of Naseerudin et al, $77-78 \%$ and only one patient was 40 years old.
[11] A study conducted in India in 2002 all patients underwent diagnostic laparoscopy to evaluate the cause of infertility .The age of the patients varied from 20-40 years. With maximum number of patients belonging 25-30 years of age. [12]Nazhat Alam (2005) whose study shows prevalence of endometriosis in primary infertility $72.27 \%$ and in secondary infertility $27.27 \%$. [13]Studies conducted in Asia and western world indicate that prevalence of is same in both classes of community. [14]

While apparently in our study the prevalence of disease is opposite to the conventional belief i.e. The poor socioeconomic class women suffered more, the opposite explanation for this difference is because of majority of the patients coming for their problem belongs to the poor socioeconomic group because of this Centre being a public sector institution. Most of the with endometriosis fall in stage I and II in our study which shows that it's not the distorted pelvic anatomy in cases of minimal and mild disease causing infertility it can be due to the changes in peritoneal environment .In similar study according to revised guideline of American Fertility society shows majority of patients in stage I and Stage II $25 \%$ and $37.5 \%$ respectively. While there were $12.55 \%$ in stage III and $25.5 \%$ in stage IV.[15]

As endometriosis is a serious gynecological issue but it is ignored. Although work has been done but still more work need to be done. The results of my study will be helpful for other health care professionals as these results can be used for further researches to identify the pathogenesis of endometriosis and cause effect relationship between endometriosis and fertility. Geographic preponderance of the disease cannot be judge .The reason is that all cases included in the study were Asian in origin.

\section{Conclusion}

Based on information from the literatures and findings of the present study we recommend recent researches and large sample size should be done in order to know the accurate figures of the endometriosis and its associated risk factors among sub fertile women undergoing diagnostic laparoscopy.

\section{References}

1. Dunselman G.A.J, Vermeulen N, Becker C, Calhaz-Jorge C, D’Hooghe T, De Bie B, et al. ESHRE guideline: Management of women with endometriosis. Human Reproduction. 2014;29(3):400-412.

2. Basmaissa, Laura O, Peter JW, Mrinal S, Hamedy S. Endometriosis and irritable bowel syndrome: a dilemma for the gynecologist and gastroenterologist The Obstetrician \&Gynaecologist. 2016; 18(1):916.

3. Tanahatoe S, Hompes PG. Lambalk CB. Accuracy of diagnostic laparoscopy in the infertility work up before intrauterine insemination. Fertilsteril . 2003;79(2):361-366.

4. Osugay, Koga K, T sulsumi O, Yano T. Maruyama M, Kugu K, et al. Role of laparoscopy in the treatment of endometriosis associated infertility. Gynecolobstet. 2002:53(Suppl 1); 93-4.

5. Imtiaz S, Zafar F, shaukata. Laparoscopic Findings in Fertility. Ann K E Med Coll. 1999; 5:93-94. 
6. Nair K,Senta F, Kamruddin A. Laparoscopic evaluation of female factors in fertility ,Med Channel. 2002;8:78-80.

7. Dunselman GAJ, Vermeulen N, Becker C, Calhaz-Jorge C, D-Hooghe T, De Bie D, et al. ESHRE guideline: management of women with endometriosis. Hum Reprod2014; 29(3):400-12.

8. Diaa M, EL-Mowafi. Laparoscopic Management of Endometriosis. Progress in Obstetrics and Gynaecology. 17TH ed. Philadelphia 2006; 343-57.

9. Tarek A, Shokeir, Heshan M. Shalan, Mohammad M, EL, Shafei. Egypt combined diagnostic approach of laparoscopy and hysteroscopy in the evaluation of female infertility result of 612 patient. J obstgynaecol Res. 2004;30(1):9-14.

10. Nakagwa K, Ohgi s, Horikawa T. Laparoscopy should be strongly considered for women with unexplained infertility. National center of Child Health and Development Abstract. 2007;33(5):665-670.
11. Naseeruddin, Khan A, Nudratullah. Prevalence and presentation of endometriosis in patients admitted in Nishtar Hospital, Multan. JAMC. 2000; 12(3):22-25.

12. Maiskarv.Desai SK, allahbadiag.Masalwalla M, Kania P. Incidence of endometriosis on laparoscopy in 230 consecutive infertility patients. J obstetgynaecol India. 2002;52 (2):99-109.

13. Nuzhatalam (2005) Dissertation,Endometriosisassociated infertility laparoscopic evaluation Bahawal Victoria Hospital,Bahawalpur.

14. Kaun Hung W, Jones GL, Vitonis A, Cramar DW, Kennedy SH, Thumas $D$ et al. Characteristics of patients with endometriosis in UK and USA, fertlsteril. 2002;78(4):767-72.

15. Hussain M, Ashraf M, Jabeen T, Nasir AK, Yasmin H, Noorani KJ. Laparoscopic evaluation of endometriosis. J Surg Pak 2004; 9:2-5. 